hep-th/9804160

HUTP-98/A035

SU-ITP-98-14

\title{
String Webs and 1/4 BPS Monopoles
}

\author{
Oren Bergman' \\ Lyman Laboratory of Physics \\ Harvard University \\ Cambridge, MA 02138 \\ Barak Kol i \\ Department of Physics \\ Stanford University \\ Stanford, CA 94305
}

April 1998

\begin{abstract}
We argue for the existence of many new 1/4 BPS states in $N=4 S U\left(N_{c}\right)$ SuperYang-Mills theory with $N_{c} \geq 3$, by constructing them from supersymmetric string webs whose external strings terminate on parallel D3-branes. The masses of the string webs are shown to agree with the BPS bound for the corresponding states in SYM. We identify the curves of marginal stability, at which these states decay into other BPS states. We find the bosonic and fermionic zero modes of the string webs, and thereby the degeneracy and spin content of some of the BPS states. States of arbitrarily high spin are predicted in this manner, all of which become massless at the conformal point. For $N_{c} \geq 4$ we find BPS states which transform in long multiplets, and are therefore not protected against becoming stable non-BPS states as moduli are varied. The mass of these extremal non-BPS states is constrained as they are connected to BPS states. Analogous geometric phenomena are anticipated.
\end{abstract}

PACS codes: 11.25.-w, 11.15.-q, 11.30.Pb

\footnotetext{
${ }^{*}$ E-mail address: bergman@string.harvard.edu

${ }^{\dagger}$ E-mail address: barak@leland.stanford.edu
} 
To the memory of Alon Cohen (1968-1997)

$\mathrm{BK}$

\section{Introduction and summary}

String theory has proven to be a powerful tool for studying various non-perturbative aspects of supersymmetric quantum field theories. D-branes have played a central role in this process, both by providing non-perturbative states in string theories (whose low energy limits are field theories) which are required by the various string dualities [1], and by providing a new test bed for quantum field theories through their world-volume dynamics [2]. D-strings in particular provide yet another insight into supersymmetric field theories, namely in determining the BPS spectrum thereof. For example, a D-string which ends on a D3-brane corresponds to a BPS monopole in the world-volume $N=4 U\left(N_{c}\right)$ SYM theory, where $N_{c}$ is the number of parallel D3-branes [3]. Similarly a bound state of $p$ fundamental strings and $q$ D-strings, where $p$ and $q$ are co-prime integers, corresponds to a $(p, q)$ BPS dyon. Such states are predicted by the conjectured $S L(2, \mathbb{Z})$ symmetry of $N=4$ SYM [ 4 ], but their existence from the field theory point of view has only been established in a limited number of cases [5]. In the string theory approach their existence follows directly from the existence of $(p, q)$ strings in type IIB string theory [6, 7]. By studying a D3-brane probe near an orientifold 7-plane, whose quantum resolution consists of two mutually non-local 7-branes 8], one can similarly construct the monopole and dyon of Seiberg-Witten theory, i.e. $N=2$ $S U(2)$ SYM, as appropriate $(p, q)$ strings between the D3-brane and either 7-brane [9].

In addition to $(p, q)$ strings, type IIB string theory contains more general stringy objects consisting of several connected $(p, q)$ strings, or "string webs" [10-19] The simplest example is a fundamental string, i.e. $(1,0)$, which terminates on a D-string, i.e. $(0,1)$, to form a "three-string junction". By charge conservation the third string must carry the charges $(1,1)$. Furthermore, unbroken supersymmetry requires the configuration to be planar, and the relative angles of the three strings to be given by $\operatorname{Arg}\left(p_{i}+q_{i} \tau\right)$, where $\tau=i / g_{s}+a, g_{s}$ is the string coupling constant, and $a$ is the expectation value of the RR scalar field. Under these conditions string webs preserve 8 of the 32 supersymmetries of type IIB string theory, and are classically static.

Just as strings ending on D-branes correspond to BPS states in the world-volume field theories, string webs whose external strings end on D-branes should correspond to BPS states, as long as the whole configuration preserves some supersymmetry. This idea has been successfully applied to understanding exceptional gauge symmetry enhancement in eight dimensions [13], finding the BPS spectrum of $5 \mathrm{~d} N=1$ gauge theories [14, 20], constructing

${ }^{1}$ Analogous $(p, q)$ 5-brane webs were constructed in [12, 14, and were used to study five-dimensional $N=1$ theories. 
1/4 BPS states in $4 \mathrm{~d} N=4 S U(3)$ SYM [21], deriving the entire BPS spectrum of SeibergWitten theory in the D3-brane probe picture [22, 23, 24], and producing $E_{8}$ flavor multiplets of quarks in theories with exceptional global symmetry [25].

In this paper we are interested in the application of general string webs to the construction of $1 / 4$ BPS states in $N=4 S U\left(N_{c}\right)$ SYM. In particular, we shall generalize the results of 21] for arbitrary $N_{c}$ and arbitrary charges. In addition, we shall determine the fermionic zero modes of the string webs, and thus the degeneracy of some of the BPS states. This will be illustrated with some simple examples.

Since any $(p, q)$ string can end on a D3-brane, it follows that string webs can end on D3-branes, and therefore give rise to new states in the world-volume field theory when the number of D3-branes is greater than two. The addition of D3-branes further limits the number of unbroken supersymmetries to 4 , so the string webs should correspond to $1 / 4$ BPS states in $N=4 S U\left(N_{c}\right)$ SYM. Such states can only appear for $N_{c} \geq 3$, which is consistent with the requirement that there be at least three D3-branes to end on. Indeed in [21] it was shown that the 3-string junction corresponds to a 1/4 BPS state in $S U(3)$ SYM carrying the charges $((1,0),(0,1))$. The mass of the 3 -string junction reproduces the BPS bound of $N=4$ SYM. Furthermore, it was shown that this state becomes marginally stable on a certain closed curve in moduli space, and that it in fact decays as the curve is crossed.

The generalization to arbitrary $N_{c}$ and arbitrary charges follows by considering more general string webs, which include more external strings, as well as internal strings. These also include degenerate string webs, in which some external strings that carry identical charges (and are therefore parallel) overlap. In this case the overlapping strings can end on the same D3-brane, and thereby give rise to non-co-prime world-volume charges. Therefore unlike 1/2 BPS states, the electric and magnetic charge pairs of $1 / 4$ BPS states need not be relatively prime. The only requirement is that the different charge pairs not have a common divisor. In that case the string web would be reducible, and therefore at most marginally stable against decay into a multi-particle state. Another kind of degeneration occurs when an external string shrinks, i.e. when one of the D3-branes coincides with a junction point. For the simple 3-string junction studied in [21] this is precisely where the state becomes marginal, and decays into the two states corresponding to the two remaining strings. The decay corresponds to separating the two strings along the common D3-brane. The same holds in the more general case; the state corresponding to the original string web becomes marginal, and decays into two other states, which correspond to the two remaining webs sharing the D3-brane in question.

The degeneracy of a BPS state which is represented by a string web, and therefore the spins of its components, is determined by the zero modes of the web [20]. The bosonic zero modes (BZM) come in two varieties, local and global. Local BZM keep the external strings fixed, whereas global BZM move them. The global BZM divide into planar and transverse modes with respect to the web, where the transverse modes correspond to translations trans- 
verse to the web, and the planar modes include the two planar translations as well as other modes. The local BZM, on the other hand, are purely planar. In the presence of D3-branes all the global planar modes and 4 of the global transverse modes are eliminated, leaving only 3 transverse modes corresponding to translations along the D3-branes, and the local BZM. The latter correspond to loops of internal strings, i.e. internal faces, which can shrink and expand without changing the mass of the web [14]. We note that in geometrical lifts to $\mathrm{M}$ theory each local BZM is expected to complexify [26, 27, 28].

To find the fermionic zero modes (FZM) one must solve the massless Dirac equation on the web. This is done by gluing together the solutions on the individual strings, i.e. constant 16 component real spinors, at the junctions and boundaries of the web. The fermionic junction and boundary constraints are found by applying the locally preserved supersymmetries to the bosonic ones. Note that although there are only 4 globally preserved supersymmetries, each junction (or boundary) actually preserves 8 locally. For a string web with $F_{\text {int }}$ internal faces and $E_{\text {ext }}$ external strings we find $n_{F Z M}=8 F_{\text {int }}+4 E_{\text {ext }}$. This is shown both by solving the FZM equations, and by generating FZM from bosonic configurations by applying different supersymmetries. The result includes FZM which were previously ignored.

Upon lifting the web to an M-theory membrane, $g=F_{\text {int }}$ is the genus, $b=E_{\text {ext }}$ is the number of boundaries, and we have $n_{F Z M}=4(2-\chi)$, where $\chi$ is the Euler character of the membrane. Using the same methods we find that for an unbounded web $n_{F Z M}=$ $8 F_{\text {int }}+8 E_{\text {ext }}$, while for a periodic web [16] $n_{F Z M}=8(F+2)$, where $F$ is the number of faces in a unit cell.

To compute the degeneracy of the state one needs to solve for the ground state of the supersymmetric quantum mechanics defined by the zero modes. For webs with no (local) BZM the quantization is straightforward, and we indeed get predictions for the degeneracy of the corresponding monopoles, and therefore for the highest spin in the supermultiplet. In the general case, even without the solution at hand, we expect that the degeneracy increases with the number of fermionic zero modes, as the spin is bounded by $\left|j_{\text {max }}\right| \leq$ $n_{F Z M} / 8=E_{\text {ext }} / 2+F_{\text {int }}$ (the bound is saturated for $F_{\text {int }}=0$ ). Since there exist webs with an arbitrary number of internal faces, there are 1/4 BPS states of arbitrary degeneracy, and therefore particles of arbitrarily high spin. All these particles will become massless when three (or more) D3-branes coincide. Indeed, having a tower of states with arbitrarily high spin becoming massless is a signature of a conformal field theory.

For $N_{c} \geq 4$ we encounter an interesting effect. As long as the D3-branes lie in a single plane, and marginal stability curves are not crossed, there exist planar webs connecting them, and therefore the states are BPS. On the other hand, planar D3-brane configurations are only a subspace of real co-dimension $4\left(N_{c}-3\right)$ of the moduli space. If we move out of this subspace the web can no longer be planar, and therefore the state cannot remain supersymmetric. BPS states thus evolve smoothly into non-BPS states as the moduli are varied. Alternatively, a non-BPS state may become BPS on a subspace of the moduli space. 
Non-BPS states have at least 16 FZM, and therefore transform in long multiplets. This would avoid contradiction with [29], if the BPS states to which they evolve would transform in long, rather than short or medium, multiplets 2 . Indeed the BPS configuration is the endpoint of non-BPS configurations, and must therefore possess at least as many fermionic zero modes. Our formula for the number of FZM confirms this. We shall refer to such BPS states as "long BPS states" or "accidental BPS states" as they exist only on a submanifold of moduli space. Despite being long, the mass of these states is protected against quantum corrections since they still preserve 4 supersymmetries.

The non-BPS state corresponding to the non-planar web will be extremal, and therefore stable ${ }^{3}$. This is realized classically as a zero force condition at each junction. Since different junctions will in general lie in different planes however, the configuration breaks supersymmetry. The classical mass of the non-BPS state can be seen to be strictly larger than the BPS bound. Furthermore, one expects quantum corrections to this mass. Since these states are continuously connected to BPS states, the quantum corrections may be studied by expanding their mass about the BPS bound as a function of parameters which measure the deviation from planarity.

It should be clarified that throughout this paper we study the string web model without performing a full quantum field theory analysis. We do not check whether the states described by the web exist as solutions of $N=4 \mathrm{SYM}$, nor that they cannot exist beyond marginal stability. Semiclassical methods may probe these questions at weak coupling, where a recently found classical solution [31] may be used as a starting point. Similar comments hold for the other results as well.

Let us point out some open questions:

- A quantum field theory analysis, perhaps using semiclassical methods, is required to check the phenomena that we describe at the string level.

- The stable non-BPS states are expected to receive quantum corrections. Being continuously connected to BPS states might facilitate such a computation.

- We found the bosonic and fermionic zero modes of a monopole represented by a web. In the general case, it remains to find the ground states of the appropriate quantum mechanics.

- All the discussed phenomena should have a geometric analog. For example, the $4 d$ $N=4$ theory can be realized as type II on $K 3 \times T^{2}$. The $K 3$ should have an $A_{N_{c}-1}$ singularity to decouple gravity, and provide the $S U\left(N_{c}\right)$ gauge group (as in geometric engineering, for example [32]). Particles would correspond to some submanifolds. The

\footnotetext{
${ }^{2}$ We thank E. Witten for suggesting the solution.

${ }^{3}$ Other examples of stable non-BPS states were discussed by Sen [30].
} 
1/4 BPS states would correspond to some special submanifolds. Marginal stability will indicate that these special submanifolds can disappear beyond some walls in moduli space, as long as they become reducible. Accidental BPS states would correspond to special submanifolds that exist only in subspaces of moduli space, and would be continuously connected to non-special minimal volume submanifolds.

The paper is organized as follows. In section 2 we review string webs and their dual grid diagrams. We also discuss degenerations, bosonic zero modes, and classification of string webs. In section 3 we generalize the results of 21] to arbitrary $N_{c}$ and arbitrary charges. We also obtain the fermionic zero modes, and discuss the classification of BPS states in $N=4$ SYM. In section 4 we discuss the smooth connection between long BPS and stable non-BPS states for $N_{c} \geq 4$.

\section{Review of string webs and grid diagrams}

In this section we shall review the construction and properties of multi-string webs and their dual grid diagrams. Most of what follows applies equally well to 5-brane webs [12, 14]. We shall use standard notation to denote the web variables: $V$ for the number of vertices, $E$ for the number of edges, and $F$ for the number of faces. These satisfy the well known Euler relation

$$
V-E+F=1 .
$$

The webs in question contain both external and internal edges, and therefore both external and internal faces. We shall denote these by $E_{\text {ext }}, E_{\text {int }}$ and $F_{\text {ext }}, F_{\text {int }}$, respectively, where $E_{\text {ext }}+E_{\text {int }}=E$ and $F_{\text {ext }}+F_{\text {int }}=F$. It is easy to see that

$$
E_{\text {ext }}=F_{\text {ext }}
$$

We shall assume that generically all vertices consist of 3 edges. This gives another relation

$$
3 V=E_{\text {ext }}+2 E_{\text {int }}
$$

If more than 3 edges meet at a point we shall interpret this as a degeneration of the web, in which some internal edges have a vanishing length.

\section{$2.1 \quad$ string webs}

A type IIB fundamental string which ends on a D-string forms a three-legged object known as a 3-string junction [10, 11]. By charge conservation, the third leg must carry the charges of both the fundamental and the Dirichlet strings, and therefore corresponds to a $(1,1)$ string. 
The condition of unbroken supersymmetry restricts the configuration to be planar, and the relative directions of the three strings in the $(x, y)$ plane to be given by

$$
e^{i \theta_{i}} \propto \Delta x_{i}+i \Delta y_{i} \propto p_{i}+q_{i} \tau,
$$

where $\tau=i / g_{s}+a, g_{s}$ is the type IIB string coupling, and $a$ is the expectation value of the $\mathrm{RR}$ scalar field. Since the tension of a $(p, q)$ string is given (in the Einstein frame) by

$$
T_{(p, q)}=\frac{1}{2 \pi \alpha^{\prime} \sqrt{g_{s}}}|p+q \tau|
$$

the above supersymmetry condition implies that the force due to the three string tensions vanishes, consistent with the idea that BPS states are stable.

Just as the fundamental string is but a single component of an infinite $S L(2, \mathbb{Z})$ multiplet of $(p, q)$ strings [6], the above configuration is only one component of a multiplet of 3-string junctions, which are all related by $S L(2, \mathbb{Z})$. All the components satisfy charge conservation,

$$
\sum_{i=1}^{3} p_{i}=\sum_{i=1}^{3} q_{i}=0,
$$

as well as the supersymmetry condition (2.4). These are the simplest examples of string webs, and in fact constitute the building blocks for all string webs.

We define an irreducible string web as a collection of $(p, q)$ strings connected by 3 -string junctions, with no disconnected components, such that the $i$ 'th string lies along $p_{i}+q_{i} \tau$. The actual orientation of the string is determined up to a change in the overall sign of $\left(p_{i}, q_{i}\right)$. The external strings are taken to be infinite, and oriented outward. The supersymmetries left unbroken by a string web oriented in the $\left(x^{8}, x^{9}\right)$ plane are given by $\epsilon_{L} Q_{L}+\epsilon_{R} Q_{R}$, with

$$
\epsilon_{L}=\Gamma_{0} \Gamma_{9} \epsilon_{L}, \quad \epsilon_{R}=-\Gamma_{0} \Gamma_{9} \epsilon_{R}, \quad \epsilon_{L}=\Gamma_{0} \Gamma_{8} \epsilon_{R}
$$

Thus string webs are invariant under $1 / 4$ of the original supersymmetry of type IIB string theory, i.e. 8 supercharges. For some simple examples see figure 1.

In the lift to M-theory string webs correspond to membranes wrapping holomorphic curves. The number of external strings is identified with the number of boundaries $E_{\text {ext }}=b$, and the number of internal faces is identified with the genus of the curve $F_{\text {int }}=g$.

\section{2 grid diagrams}

A grid diagram (similar diagrams appear in toric geometry) is defined on a $2 \mathrm{~d}$ square integer lattice. We shall denote its components by points, lines and polygons. The diagram consists of points, which lie on the grid, and of lines joining them. The contour of the diagram is 


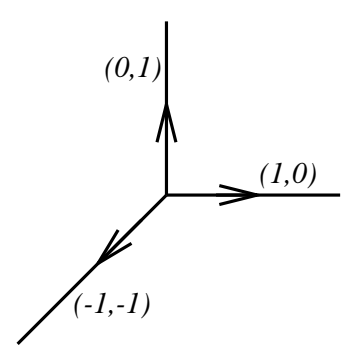

a

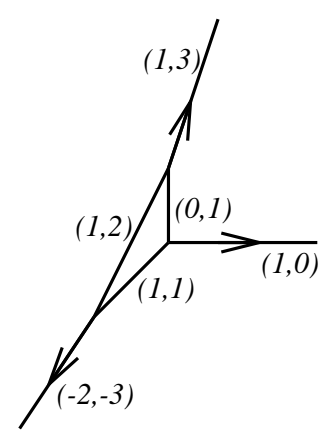

c

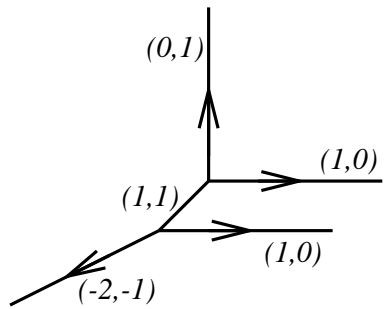

b

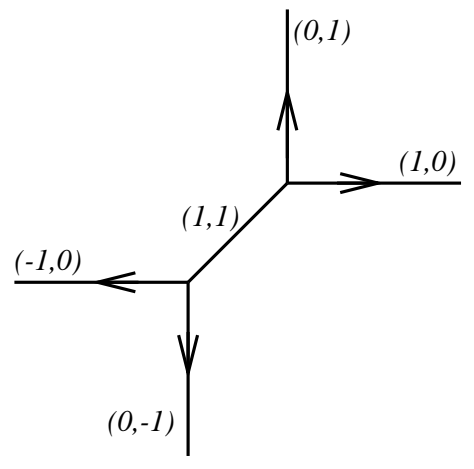

$\mathrm{d}$

Figure 1: Examples of simple string webs: (a) a 3-string junction, (b) a web with an internal string, (c) a web with an internal face, (d) another web with an internal string.

convex, and so are its internal polygons. There may be more conditions on the diagram, but rather than state all of them, we shall describe how to build it.

To each string web one can associate a dual grid diagram, by exchanging faces with points, $(p, q)$ edges (strings) with $\pm(-q, p)$ lines, and vertices (junctions) with polygons. Consequently, the grid diagram consists of $V$ adjacent polygons, and has $F_{\text {ext }}$ edge points, $F_{\text {int }}$ internal points, $E_{\text {ext }}$ external lines, and $E_{\text {int }}$ internal lines. The grid diagrams dual to the four string webs of figure 1 are shown in figure 2 .

To construct a grid diagram one starts by marking an arbitrary point on the grid which is chosen to correspond to some face in the web. Crossing to an adjacent face requires passing through a $(p, q)$ string, and thus an orthogonal line represented by the grid vector $\pm(-q, p)$ should be marked, ending at a point that represents the adjacent face. Consistency requires that if we go around a closed loop of faces in the web, circling a junction, we will return to the same point in the diagram. This is guaranteed by the charge conservation property of the junction (2.6). Convexity can be derived by going around infinity in the web. 


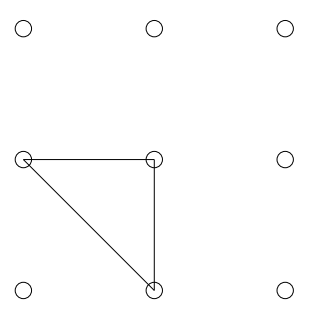

$\mathrm{a}$

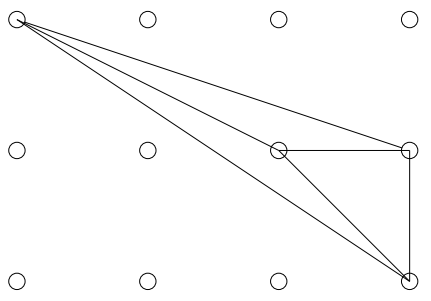

C

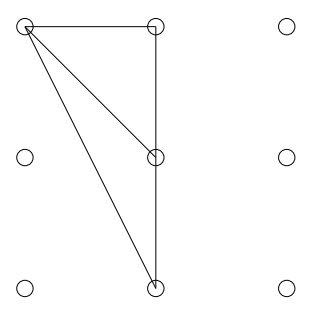

b

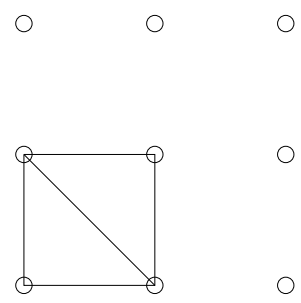

d

Figure 2: Grid diagrams dual to the webs in figure 1. Note that (b) is a quadrangle.

\section{3 degenerations}

It may be the case that some of the internal edges of a web have a vanishing length. We shall refer to this as a degeneration of the web. In some cases degenerations may have interesting physical consequences, for example:

- External (figure 1b) or internal edges may overlap. In this case a web with $E_{\text {ext }}$ external ( $E_{\text {int }}$ internal) edges degenerates into what looks like a web with less than $E_{\text {ext }}$ external ( $E_{\text {int }}$ internal) edges, where some of them have non-co-prime $(p, q)$ charges. In the dual grid diagram such a potential degeneration corresponds to a point in the interior of an external (internal) line, e.g. figure 2b. For 5-brane webs, overlapping external and internal edges correspond to enhanced global and gauge symmetries, respectively, in the five dimensional field theories [12, 14]. For string webs, overlapping external edges will be relevant when we discuss $1 / 4$ BPS states in $N=4 S U\left(N_{c}\right) \mathrm{SYM}$, since they can end on the same D3-brane, and thus give rise to non-co-prime world-volume charges.

- An internal face may shrink, e.g. figure 1c. For 5-brane webs this corresponds to a fixed point of the renormalization group in the five-dimensional field theory.

- A web may become reducible, e.g. figure 1d, and therefore only marginally bound. For 
5-brane webs this would be a flop transition in the corresponding Calabi-Yau.

If the external strings of a string web are not infinite, but rather terminate on D-branes (specifically D3-branes), there will be another kind of degeneration, corresponding to shrinking external strings. At such a degeneration, the string web becomes marginal, and in fact decays into smaller webs. This will be explained in section 3 .

\section{4 bosonic zero modes}

A bosonic zero mode (BZM) is an infinitesimal deformation of the web which does not change its mass (see [33] for a related study of the string web worldsheet). Equivalently it is a solution of the scalar Laplace equation on the web. Solutions on the entire web can be constructed by gluing together the solutions for the individual edges (strings) at the vertices (junctions). The latter are given by a constant 8-vector $X$ on each string (we do not need to consider rotations here). These decompose into 7-vectors normal to the plane of the web, $X_{N}$, and scalars tangent to the plane of the web (and normal to the string) $X_{T}$,

$$
X \rightarrow X_{N}+X_{T}
$$

These variables are constrained at the junctions by

$$
\begin{aligned}
X_{N}^{1}=X_{N}^{2} & =X_{N}^{3} \\
\sum_{i=1}^{3}( \pm)\left|p_{i}+\tau q_{i}\right| X_{T}^{i} & =0
\end{aligned}
$$

where the superscript $i=1,2,3$ labels the strings that meet at the junction. The sign is determined by whether the planar deformation of a given string is clockwise or counterclockwise with respect to the junction.

The condition (2.9) implies that the web has a total of 7 normal BZM. These correspond to the motion of the entire web in the transverse directions. The condition (2.10) gives $V$ (independent) junction constraints for $E$ variables. Thus the number of planar BZM is $E-V=F-1=F_{\text {int }}+E_{\text {ext }}-1$, where we have used (2.1) and (2.2).

We distinguish between local BZM and global BZM. Define the support of a zero mode to be the union of all strings on which the mode does not vanish. A local BZM does not move the external strings, so these are not included in its support. The number of global BZM is $E_{\text {ext }}-1$, which includes the two translations in the plane of the web. From the counting we see that each internal face contributes one local BZM, which is realized as the shrinking/expansion mode of that face. In general, the web might have "hidden faces", and one should instead count the internal points in the dual grid diagram.

By supersymmetry, string webs possess fermionic zero modes (FZM) as well. Furthermore, if the external strings terminate on D3-branes, the number of both BZM and FZM will change due to the boundary conditions. This will be shown in section 3 . 


\section{5 classification of string webs}

As we have mentioned, the simple 3-string junction (figure 1a) is one component of an $S L(2, \mathbb{Z})$ multiplet of 3 -string junctions. Likewise all string webs belong to $S L(2, \mathbb{Z})$ multiplets. These can be classified using $S L(2, \mathbb{Z})$ invariants of string webs. The obvious invariants are the web variables $V, E_{\text {ext,int }}$, and $F_{\text {ext,int }}$. These are however not independent, as they are related by (2.1),(2.2), and (2.3). The two independent invariants can be taken to be $E_{\text {ext }}$ and $F_{\text {int }}$.

Consider for example string webs with $E_{\text {ext }}=3$. These are generalizations of the 3 -string junction, to include an arbitrary number of internal faces $F_{\text {int }}$. Label the external strings $\left(p_{1}, q_{1}\right),\left(p_{2}, q_{2}\right)$, and $\left(p_{3}, q_{3}\right)$. By charge conservation $p_{3}=-p_{1}-p_{2}$ and $q_{3}=-q_{1}-q_{2}$. One can define an $S L(2, \mathbb{Z})$ invariant intersection number 34

$$
\mathcal{I}=\left|\begin{array}{cc}
p_{1} & p_{2} \\
q_{1} & q_{2}
\end{array}\right|=p_{1} q_{2}-p_{2} q_{1}
$$

This is simply twice the (oriented) area of the triangle in the corresponding grid diagram, and is related to the number of internal points $F_{\text {int }}$ as follows

$$
\mathcal{I}=2 A=2\left(F_{\text {int }}+1 / 2\right)
$$

We shall refer to this as an "area" type invariant. For webs with more external strings there will be several "area" invariants, given by the areas of the triangular sub-polygons, modulo constraints. For example, webs with $E_{\text {ext }}=4$ have dual grid diagrams with four external lines, i.e. quadrangles f. These can be divided into two "triangles" in two ways, corresponding to the two diagonals [? Thus there are four "triangular" sub-polygons with areas $A_{1}, \ldots, A_{4}$. There is one constraint coming from the one quadrangle, namely $A_{1}+$ $A_{2}=A_{3}+A_{4}$. That leaves three invariants, which can be related to the three independent intersection numbers

$$
\mathcal{I}_{12}=\left|\begin{array}{cc}
p_{1} & p_{2} \\
q_{1} & q_{2}
\end{array}\right| \quad \mathcal{I}_{23}=\left|\begin{array}{cc}
p_{2} & p_{3} \\
q_{2} & q_{3}
\end{array}\right| \quad \mathcal{I}_{31}=\left|\begin{array}{cc}
p_{3} & p_{1} \\
q_{3} & q_{1}
\end{array}\right|
$$

This generalizes to arbitrary values of $E_{\text {ext }}$. The number of independent intersection numbers is given by

$$
\left(\begin{array}{c}
E_{e x t}-1 \\
2
\end{array}\right)
$$

\footnotetext{
${ }^{4}$ For an $E_{\text {ext }}$ sided polygon with $F_{\text {int }}$ internal points, the area is given by $A=F_{\text {int }}+E_{\text {ext }} / 2-1$.

${ }^{5}$ These may be degenerate, as in figure $2 \mathrm{~b}$.

${ }^{6}$ If the diagonals go through internal points, these "triangles" may actually be higher polygons.
} 
In the grid diagram this is understood as follows. One counts the number of triangular sub-polygons and subtracts the number of constraints. These are given by the number of quadrangular sub-polygons, minus constraints on constraints, etc. The result is

$$
\left(\begin{array}{c}
E_{e x t} \\
3
\end{array}\right)-\left(\begin{array}{c}
E_{e x t} \\
4
\end{array}\right)+\left(\begin{array}{c}
E_{e x t} \\
5
\end{array}\right)-\cdots=\sum_{k=3}^{E_{e x t}}(-1)^{k+1}\left(\begin{array}{c}
E_{e x t} \\
k
\end{array}\right) .
$$

One can then check using the binomial formula that this is indeed equal to (2.14).

There are additional invariants which are not related to areas of polygons (or intersection numbers). For the case $E_{\text {ext }}=3$ there is one such invariant, which is understood as follows [34. Using $S L(2, \mathbb{Z})$ we can transform one of the external strings into a $(1,0)$ string. The charges of the external strings are then given by

$$
(1,0), \quad(p, q), \quad(-p-1,-q)
$$

The integer $q$ is equal to the "area" invariant $\mathcal{I}$, and its value is arbitrary. The other invariant is equal to the integer $p$, whose value determines the shape of the grid diagram (see figure 3). Hence it is referred to as a "shape" invariant. There are residual $S L(2, \mathbb{Z})$ transformations which preserve the $(1,0)$ string. These are given by

$$
\left(\begin{array}{ll}
1 & n \\
0 & 1
\end{array}\right), \quad n \in \mathbb{Z} .
$$

Under such a transformation $p \rightarrow p+n q$, so only values of $p$ in $[0, q-1]$ are $S L(2, \mathbb{Z})$ inequivalent. The allowed values of $p$ are actually more restricted, since both $(p, q)$ and $(-p-1,-q)$ must be co-prime. For $q>0$ and prime, the only excluded values are $p=0$ and $p=q-1$. For $q$ non-prime there will be additional exclusions. In the grid diagram the exclusions can be understood as the appearance of additional edge points as the shape of the diagram is changed (figure 3 ).

\section{$3 \quad 1 / 4$ BPS States in $S U\left(N_{c}\right)$ SYM}

In this section we will generalize the results of [21] to 1/4 BPS states of $S U\left(N_{c}\right)$ SYM for arbitrary $N_{c}$, carrying arbitrary electric and magnetic charges, and with an arbitrary value of $\theta_{Y M}$. States in $S U\left(N_{c}\right)$ SYM are characterized in part by their $\left(N_{c}-1\right)$-dimensional electric and magnetic charge vectors $(\mathbf{p}, \mathbf{q})$. These charges are related via the vacuum expectation value of the sextuplet Higgs field in the Cartan subalgebra $\langle\overrightarrow{\boldsymbol{\Phi}}\rangle=\overrightarrow{\mathbf{v}}$ to six-dimensional 


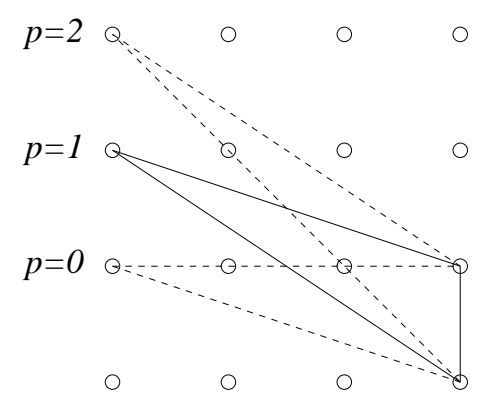

Figure 3: Grid diagrams with $E_{\text {ext }}=3, q=3$ and varying shapes. The cases $p=0,2$ are excluded as they contain new edge points, and therefore really have $E_{\text {ext }}>3$.

electric and magnetic charge vectors, given by \

$$
\vec{Q}_{E}=g_{Y M} \sum_{a=1}^{N_{c}-1}\left(p^{a} \boldsymbol{\beta}^{(a)} \cdot \overrightarrow{\mathbf{v}}+\frac{\theta_{Y M}}{2 \pi} q^{a} \boldsymbol{\beta}^{(a) *} \cdot \overrightarrow{\mathbf{v}}\right) \quad, \quad \vec{Q}_{M}=\frac{4 \pi}{g_{Y M}} \sum_{a=1}^{N_{c}-1} q^{a} \boldsymbol{\beta}^{(a) *} \cdot \overrightarrow{\mathbf{v}},
$$

where $\boldsymbol{\beta}^{(a)}$ and $\boldsymbol{\beta}^{(a) *}\left(a=1, \ldots, N_{c}-1\right)$ are the simple roots and simple co-roots, respectively, of $S U\left(N_{c}\right)$. Since $S U\left(N_{c}\right)$ is simply-laced $\boldsymbol{\beta}^{(a)}=\boldsymbol{\beta}^{(a) *}$. The central charges of the $N=4$ superalgebra are given by 35

$$
Z_{ \pm}^{2}=\left|\vec{Q}_{E}\right|^{2}+\left|\vec{Q}_{M}\right|^{2} \pm 2\left|\vec{Q}_{E} \times \vec{Q}_{M}\right|
$$

and therefore the BPS bound on the mass of charged states is

$$
M_{(\mathbf{p}, \mathbf{q})} \geq Z_{+} .
$$

There are two possible kinds of BPS states. The $W$-bosons, monopoles, and usual dyons have $\mathbf{p} \propto \mathbf{q}$, and therefore $\vec{Q}_{E} \propto \vec{Q}_{M}$. These states preserve one half of the underlying supersymmetry, i.e. eight supercharges, and transform in a short $\left(2^{4}\right)$ representation of the superalgebra, with $j_{\max }=1$ (vector multiplets). Hence they are referred to as $1 / 2$ BPS states. On the other hand, if $N_{c}>2$, it is possible to have $\mathbf{p} \propto \mathbf{q}$, and therefore $\vec{Q}_{E} \not \propto \vec{Q}_{M}$. Such states would preserve only one quarter of the supersymmetry, i.e. four supercharges, and therefore transform in a medium $\left(2^{6}\right)$ representation of the superalgebra, with $j_{\max }=3 / 2$. We refer to these as $1 / 4$ BPS states.

\section{1 geometry and BPS mass}

In the D-brane construction we have $N_{c}$ parallel D3-branes, with positions $\vec{R}_{a}$ in the transverse $\mathbb{R}^{6}$ space. This gives a world-volume gauge group $U\left(N_{c}\right) \sim S U\left(N_{c}\right) \times U(1)$. The $U(1)$

\footnotetext{
${ }^{7}$ We shall use boldface to denote $\left(N_{c}-1\right)$-dimensional vectors, and an $\overrightarrow{a r r o w}$ to denote six-dimensional vectors.
} 
factor corresponds to the c.o.m. degrees of freedom which we can ignore. Let us fix the position of one of the D3-branes at the origin $\vec{R}_{N_{c}}=0$, and parameterize the moduli space by the positions of the $N_{c}-1$ others. This also means that we will parameterize the Cartan subalgebra of $S U\left(N_{c}\right)$ according to the $N_{c}-1$ free D3-branes.

The SYM coupling constant and theta angle are related to the string coupling constant and RR scalar as

$$
\tau=\frac{4 \pi i}{g_{Y M}^{2}}+\frac{\theta_{Y M}}{2 \pi}=\frac{i}{g_{s}}+a
$$

The positions of the D3-branes are related to VEV's of the Higgs field as follows

$$
\vec{R}_{a}=4 \pi^{3 / 2} \alpha^{\prime} \boldsymbol{\beta}^{(a)} \cdot \overrightarrow{\mathbf{v}}
$$

A simple way to determine the precise numerical factor is to compare the masses of the $W$-boson and monopole to the masses of a $(1,0)$ string and a $(0,1)$ string between two D3-branes. We shall henceforth set $2 \pi \alpha^{\prime}=1$, and assume that $g_{s}=1$ and $a=0$. The generalization to other values is achieved by simply replacing

$$
\mathbf{p} \longrightarrow g_{s}(\mathbf{p}+a \mathbf{q})
$$

We can now express the central charge $Z_{+}$in terms of the D3-brane positions $\vec{R}_{a}$ :

$$
Z_{+}^{2}=\sum_{a, b=1}^{N_{c}-1}\left(p^{a} p^{b}+q^{a} q^{b}\right) \vec{R}_{a} \cdot \vec{R}_{b}+2\left|\sum_{a, b=1}^{N_{c}-1} p^{a} q^{b} \vec{R}_{a} \times \vec{R}_{b}\right|
$$

We shall assume that $\mathbf{p}$ and $\mathbf{q}$ have no common vanishing components. If they did, the problem would reduce to finding BPS states in $S U\left(N_{c}-k\right)$, where $k$ is the number of common vanishing components. We are therefore interested in configurations where strings end on all $N_{c}$ D3-branes.

Let us first consider degenerate string webs, in which all internal strings have vanishing length, and therefore all the external strings meet at a point (figure 4a). We will then generalize to include non-vanishing internal strings (figure $4 \mathrm{~b}$ ), as well as non-vanishing internal faces (figure $4 \mathrm{c}$ ). The external strings carry charges $\left(p_{1}, q_{1}\right), \ldots,\left(p_{N_{c}}, q_{N_{c}}\right)$, and lie along the vectors $\vec{A}_{a}\left(a=1, \ldots, N_{c}\right)$. The geometrical relations that follow from figure $4 \mathrm{a}$ are given by

$$
\left|\vec{R}_{a}-\vec{R}_{b}\right|^{2}=\left|\vec{A}_{a}-\vec{A}_{b}\right|^{2}=A_{a}^{2}+A_{b}^{2}-2 A_{a} A_{b} \cos \theta_{a b} \quad\left(a, b=1, \ldots, N_{c}\right),
$$

where $\theta_{a}=\operatorname{Arg}\left(\vec{A}_{a}\right)$, and $\theta_{a b} \equiv \theta_{a}-\theta_{b}$. The supersymmetry condition (2.4) implies that (with $g_{s}=1$ and $a=0$ )

$$
\tan \theta_{a}=\frac{q_{a}}{p_{a}}
$$


and therefore that

$$
\cos \left(\theta_{a}-\theta_{b}\right)=\frac{p_{a} p_{b}+q_{a} q_{b}}{\sqrt{\left(p_{a}^{2}+q_{a}^{2}\right)\left(p_{b}^{2}+q_{b}^{2}\right)}} \quad, \quad \sin \left(\theta_{a}-\theta_{b}\right)=\frac{p_{b} q_{a}-p_{a} q_{b}}{\sqrt{\left(p_{a}^{2}+q_{a}^{2}\right)\left(p_{b}^{2}+q_{b}^{2}\right)}} .
$$

Using (3.8) and (3.10) in (3.7) we find

$$
Z_{+}=\sum_{a=1}^{N_{c}} A_{a} \sqrt{\left(p_{a}^{2}+q_{a}^{2}\right)}
$$

where $p_{N_{c}}=-\sum_{a=1}^{N_{c}-1} p_{a}$, and similarly for $q_{N_{c}}$. This is precisely the mass of the string web in figure $4 \mathrm{a}$.

For string webs that have internal strings, but no (finite size) internal faces, a generic geometry is shown in figure $4 \mathrm{~b}$. The internal strings carry charges $\left(r_{1}, s_{1}\right), \ldots,\left(r_{E_{i n t}}, s_{E_{\text {int }}}\right)$, and lie along the vectors $\vec{B}_{\alpha}\left(\alpha=1, \ldots, E_{\text {int }}\right)$. Generically, a path from one D3-brane to another goes through $k$ internal strings $\vec{B}_{\alpha_{1}}, \ldots, \vec{B}_{\alpha_{k}}$, so the geometrical relations are now

$$
\left|\vec{R}_{a}-\vec{R}_{b}\right|^{2}=\left|\vec{A}_{a}-\vec{A}_{b}+\sum_{i=1}^{k}( \pm) \vec{B}_{\alpha_{i}}\right|^{2}
$$

where the sign depends on the orientation of the vector $\vec{B}_{\alpha_{i}}$ relative to the path. The supersymmetry condition for the internal strings is

$$
\tan \phi_{\alpha}=\frac{s_{\alpha}}{r_{\alpha}}
$$

where $\phi_{\alpha}=\operatorname{Arg}\left(\vec{B}_{\alpha}\right)$, and there are therefore relations analogous to (3.10) for $\left(\phi_{\alpha}-\phi_{\beta}\right)$ and $\left(\theta_{a}-\phi_{\beta}\right)$. These, together with (3.12), give

$$
Z_{+}=\sum_{a=1}^{N_{c}} A_{a} \sqrt{p_{a}^{2}+q_{a}^{2}}+\sum_{\alpha=1}^{E_{\text {int }}} B_{\alpha} \sqrt{r_{\alpha}^{2}+s_{\alpha}^{2}}
$$

which is precisely the mass of the string web.

Finally, in the most general case string webs can have internal faces as well (figure 4c). The geometrical relations are still (3.12), but the steps to (3.14) are a little more complicated, as some internal strings may not appear in any paths. On the other hand, we already know that shrinking an internal face of a string web corresponds to a bosonic zero mode (we will see that this persists in the presence of D3-branes as well in subsection 3.3), so the mass of the web is independent of this deformation. Since shrinking the internal faces to zero size reduces the web to the previous case, the agreement holds in the presence of finite internal faces as well, and therefore for generic string webs. 


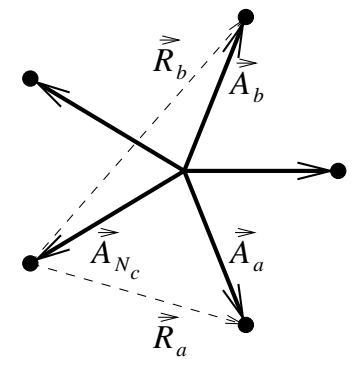

a

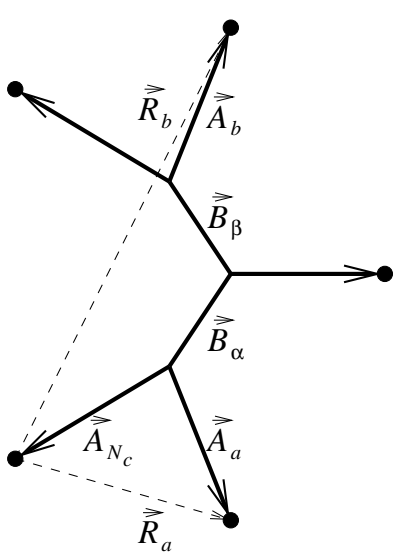

$\mathrm{b}$

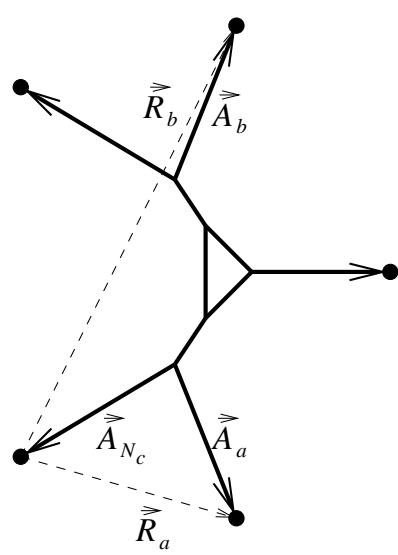

$\mathrm{C}$

Figure 4: 1/4 BPS states from string webs: (a) completely degenerate web, (b) web without internal faces, (c) generic web.

Note that the agreement holds regardless of whether the charges $p_{a}$ and $q_{a}$ are co-prime or not, i.e. whether some external strings are overlapping. We expect a $1 / 4$ BPS state in the field theory (modulo marginal stability, soon to be addressed) for all sets $\left\{\left(p_{a}, q_{a}\right)\right\}$ of charges satisfying $\sum^{N_{c}} p_{a}=\sum^{N_{c}} q_{a}=0$, as long as the charges of the different external strings do not have a common divisor. If there is a common divisor, say $k$, the configuration is reducible to $k$ irreducible ones, corresponding to $k 1 / 4$ BPS states, and the state could be at most marginally bound.

An alternative derivation for the equivalence of the BPS formula and the mass of the web is given in Appendix A.

\section{2 examples}

Let us consider a few examples of $1 / 4$ BPS states in $S U(3)$ and $S U(4)$ SYM. We shall use the four examples of string webs from the previous section (figure 1), and assume that the external strings terminate on D3-branes.

Example 1 The 3 -string junction in figure 1a corresponds to the state $\left(\left(p_{1}, q_{1}\right),\left(p_{2}, q_{2}\right)\right)=$ $((1,0),(0,1))$ in $S U(3)$, which was studied in [21].

Example 2 The web in figure 1 b has four external strings, $(0,1),(-2,-1)$, and two (1,0)'s. When these end on four D3-branes a $1 / 4$ BPS state with charges $((0,1),(1,0),(1,0))$ results in the world-volume $S U(4)$ theory. In the degenerate case, i.e. when the internal $(1,1)$ string shrinks, the two $(1,0)$ strings coincide, and can therefore terminate 
on a single D3-brane. This gives rise to a $1 / 4$ BPS state in $S U(3)$, with charges $((0,1),(2,0))$.

Example 3 The web in figure 1c has three external strings and a single internal face. It gives rise to a $1 / 4$ BPS state in $S U(3)$ SYM with charges $((1,0),(1,3))$. This state has a single bosonic zero mode, which must be quantized.

Example 4 The web in figure 1d has four external strings, and therefore gives rise to a 1/4 BPS state in $S U(4)$ SYM. The charges of this state are $((1,0),(0,1),(0,-1))$.

All of the above states preserve $1 / 4$ of the underlying supersymmetry of $N=4$ SYM, and therefore would seem to transform in medium sized $\left(2^{6}\right)$ multiplets, with $j_{\max }=3 / 2$. However, as we shall soon see, there are generically additional fermionic zero modes beyond those corresponding to the 12 broken supersymmetries. These generate additional degeneracy, and thus increase the size of the multiplets, and with it $j_{\max }$.

\section{3 fermionic zero modes}

In section 2 we analyzed the bosonic zero modes (BZM) of string webs. Here we shall first extend the discussion of the BZM to include D3-brane boundaries, and then we shall find the fermionic zero modes (FZM).

Adding the D3-branes further decomposes $X_{N}$, the deformation 7-vector normal to the web, into a 4 -vector perpendicular to the D3-branes, $X_{N \perp}$, and a 3 -vector parallel to the branes, $X_{N \|}$

$$
X_{N} \rightarrow X_{N \perp}+X_{N \|}
$$

The boundaries constrain the strings ending on the D3-branes, i.e. the external strings, to move only in worldvolume directions, so

$$
\left.X_{T}\right|_{e x t}=\left.X_{N \perp}\right|_{e x t}=0 .
$$

This eliminates the planar global BZM, leaving only the $F_{\text {int }}$ local BZM. It also eliminates 4 of the 7 normal BZM, leaving 3 which account for translations along the D3-branes.

The local BZM actually need to be complexified to give the full smooth moduli space of the web. Though we will not use this fact, note that in a web description there is a singularity (or boundary) in the moduli space when an internal face expands until it hits a D3-brane. Experience with geometrical descriptions of the system [26, 27, 28] teaches us that each modulus in the web picture could be paired with a complex phase.

Let us now derive the equations for the fermionic zero modes (FZM). As for the BZM, we can solve the Dirac equation on the web by gluing together the solutions on individual strings. The latter are constant 16 component spinors $\Psi$, which transform in the same 
representations as the broken supersymmetries of the respective strings. We would like to describe the constraints on these variables at the junctions and at the boundaries. The spinors decompose under the transverse $S O(7)$ subgroup as

$$
\Psi \rightarrow \Psi_{T}+\Psi_{N}
$$

where $\Psi_{T}, \Psi_{N}$ are the superpartners of $X_{T}, X_{N}$, respectively, under the 8 supersymmetries preserved by the web (the number of bosons and fermions in a multiplet does not have to match as the zero modes are quantum mechanical, rather than field theoretic, variables.).

In the presence of D3-branes only 4 supersymmetries are unbroken. However, since junctions still preserve the original 8 supersymmetries locally, one can derive the fermionic junction conditions by acting on the bosonic ones (2.9), (2.10) with these supersymmetries:

$$
\begin{array}{r}
\Psi_{N}^{1}=\Psi_{N}^{2}=\Psi_{N}^{3} \\
\sum_{i=1}^{3}( \pm)\left|p_{i}+\tau q_{i}\right| \Psi_{T}^{i}=0 .
\end{array}
$$

We use the same sign conventions as in the bosonic case.

After introducing the D3-branes the spinors are further decomposed under $S O(7) \rightarrow$ $S O(3) \times S O(4)$ as

$$
\begin{aligned}
& \Psi_{T} \rightarrow(\mathbf{2},(\mathbf{2}, \mathbf{1}))+(\mathbf{2},(\mathbf{1}, \mathbf{2}))=\Psi_{T \perp}+\Psi_{T \|} \\
& \Psi_{N} \rightarrow(\mathbf{2},(\mathbf{2}, \mathbf{1}))+(\mathbf{2},(\mathbf{1}, \mathbf{2}))=\Psi_{N \|}+\Psi_{N \perp},
\end{aligned}
$$

where $\Psi_{T \perp}, \Psi_{N \perp}$, and $\Psi_{N \|}$ are the superpartners of $X_{T}, X_{N \perp}$, and $X_{N \|}$, respectively, under the 4 globally preserved supersymmetries $\left(\Psi_{T \|}\right.$ has no superpartner). At a given boundary there are 8 locally preserved supersymmetries (supersymmetries that are preserved both by the D3-brane and the string ending on it). By acting with these supersymmetries on the bosonic boundary constraint (3.16) we get the fermionic boundary constraint 5 :

$$
\left.\Psi_{T \perp}\right|_{e x t}=\left.\Psi_{N \perp}\right|_{e x t}=0
$$

Having derived the equations for the FZM we would like to describe their solutions. First we shall count the solutions directly from the equations, then we shall confirm the result by generating FZM from BZM by acting with supersymmetries. Equations (3.18) and (3.21) imply that $\Psi_{N \perp}=0$ on all the strings. On the other hand, the solution $\Psi_{N \|}=$ const (same constant on all the strings) describes 4 fermionic zero modes. For $\Psi_{T \|}$ there are $4 E$ variables and $4 V$ (independent) constraints from (3.19), which gives $4(E-V)=4\left(F_{\text {int }}+E_{\text {ext }}-1\right)$ FZM. For $\Psi_{T \perp}$, on the other hand, there are only $4 E_{i n t}$ variables, since the ones on the

\footnotetext{
${ }^{8}$ Actually it is sufficient in this case to act only with the 4 globally preserved supersymmetries.
} 
external strings must vanish (3.21). Eq. (3.19) gives $4 V$ constraints on $\Psi_{T \perp}$, but they are not independent. The constraints are related by a single (spinor) equation. This can be seen by summing the constraints in (3.19) over all the vertices, and noting that the contributions of internal strings drop out since they always contribute to two vertices with opposite sign, and that the contribution of the external strings vanishes due to (3.21). There are therefore $4(V-1)$ independent constraints on the $4 E_{\text {int }}$ variables, leaving $4\left(E_{\text {int }}-V+1\right)=4 F_{\text {int }}$ FZM. Summing all the contributions gives a total of $8 F_{\text {int }}+4 E_{\text {ext }}$.

The FZM can also be obtained directly from the BZM using certain supersymmetry (not necessarily unbroken) transformations. This method has the advantage of giving the metric on moduli space. Consider first a local BZM. The support of such a mode does not include external strings, which end on D3-branes, and therefore it preserves 8 supersymmetries. By applying these to the BZM we get constant fermionic variables that satisfy the junction constraint (3.19) by construction. Since there are $F_{\text {int }}$ local BZM, the above procedure produces $8 F_{\text {int }}$ FZM 9 . Similarly, one can obtain the $4 \mathrm{FZM}$ corresponding to $\Psi_{N \|}=$ const by acting on the translational BZM $X_{N \|}=$ const with the 4 globally preserved supersymmetries.

The remaining FZM can be generated from the $E_{\text {ext }}-1$ planar global BZM of the unbounded web (section 2.4), even though they no longer exist in the presence of D3-branes. Let us act on these BZM, i.e. $X_{T}$ with $\left.X_{T}\right|_{\text {ext }} \neq 0$, with the 4 supersymmetries which are preserved by the web, but broken by the D3-branes. This produces constant fermionic variables which satisfy the junction conditions. Furthermore, as these supersymmetries transform in the $(\mathbf{2},(\mathbf{1}, \mathbf{2}))$ of $S O(3) \times S O(4)$, we obtain $\Psi_{T \|}$ rather than $\Psi_{T \perp}$. The boundary condition $\left.\Psi_{T \perp}\right|_{\text {ext }}=0$ can therefore be satisfied despite the fact that $\left.X_{T}\right|_{\text {ext }} \neq 0$. Since both the junction and boundary conditions are satisfied, these are indeed FZM, and there are $4\left(E_{\text {ext }}-1\right)$ of them. The total number of FZM is then

$$
n_{F Z M}=8 F_{\text {int }}+4+4\left(E_{\text {ext }}-1\right)=8 F_{\text {int }}+4 E_{\text {ext }},
$$

in agreement with the previous count.

An advantage of this method of generating FZM is that the fermionic mass matrix (the metric on the fermionic coordinates of moduli space) is automatically determined. The mass of an FZM is given by the mass of the BZM that was used to generate it. For BZM, the metric on moduli space $g_{i j}$ can be read off from the expression for the kinetic energy

$$
g_{i j}=\sum_{a \in \text { strings }} m_{a} X_{a i} X_{a j}
$$

where $i, j$ label the BZM. The fermionic kinetic term is analogously $g_{i j}=\sum m_{a} \bar{\Psi}_{a i} \Psi_{a j}$. We see here explicitly that FZM generated from BZM will have the same metric. This applies

\footnotetext{
${ }^{9}$ Another way to see that each local BZM gives 8 FZM is to consider a 5 -brane $(p, q)$ web, where each face is known to give rise to a vector multiplet [14. A vector multiplet in $5 \mathrm{~d}$ has one scalar, which is the BZM, 4 fermionic degrees of freedom coming from 8 fermionic variables, which are the 8 FZM, and 3 vector degrees of freedom which do not appear in the quantum mechanics.
} 
not only to FZM generated by locally unbroken supersymmetries, but also to the FZM that are generated by broken supersymmetries.

Let us illustrate the result in (3.22) by some examples:

- The $W$ boson is just an open string, and therefore has $E_{\text {ext }}=2, F_{\text {int }}=0$. Eq. (3.22) gives 8 FZM, which is consistent with it being $1 / 2$ BPS and preserving 8 supersymmetries. The FZM generate a $2^{4}$ (short) representation of the superalgebra with $j \leq 1$.

- The 3-string junction (figure 1a) has $E_{\text {ext }}=3, F_{\text {int }}=0$, and therefore $12 \mathrm{FZM}$, which agrees with the number of broken supersymmetries. These generate a $2^{6}$ (medium) representation with $j \leq 3 / 2$.

- The states arising from the string webs in figures $1 \mathrm{~b}, \mathrm{~d}$ have $E_{\text {ext }}=4, F_{\text {int }}=0$, and therefore 16 FZM, 4 of which do not correspond to broken supersymmetries. These generate a $2^{8}$ representation with $j \leq 2$.

- The state in figure $1 \mathrm{c}$ has $E_{\text {ext }}=3, F_{\text {int }}=1$, and therefore $20 \mathrm{FZM}$. The 4 modes related (by supersymmetry) to translations along the D3-branes have masses which are independent of the bosonic moduli, while the masses of the other modes depend on the size of the internal face, as can be seen for the corresponding BZM. The former generate a $2^{2}$ representation, which is tensored with the ground states of the quantum mechanics on a 1 complex dimensional manifold with the remaining 16 FZM.

One can also use the above methods to count fermionic zero modes of string webs with no D3-brane boundaries. One gets

web without boundaries: $n_{F Z M}=8 F_{\text {int }}+8+8\left(E_{\text {ext }}-1\right)=8 F_{\text {int }}+8 E_{\text {ext }}$,

where $8 F_{\text {int }}$ are obtained from local BZM, 8 are obtained from translations in the 7 transverse directions, and $8\left(E_{\text {ext }}-1\right)$ are obtained from planar global BZM.

For periodic string webs [16] (a net on a torus), the BZM consist of 7 translations transverse to the web, and $F+1$ modes in the plane of the web, two of which are translations, where $F$ is the number of faces in a unit cell. Varying the translations with respect to the 8 supersymmetries preserved by the web gives $8 \mathrm{FZM}$, and varying the planar modes gives $8(F+1)$ FZM. The former are the solutions of the $\Psi_{N}$ equations (3.18), and the latter solve the $\Psi_{T}$ equations (3.19). Summing the FZM gives

$$
\text { web on torus: } n_{F Z M}=8(F+2) \text {. }
$$

In the lift to M-theory the string web becomes a smooth membrane, with genus $g=F_{\text {int }}$ and $b=E_{\text {ext }}$ boundaries. Fermionic zero modes correspond to zero eigenvalues of the Dirac operator on the membrane. For a web with D3-brane boundaries we have $n_{F Z M}=4(2-\chi)$, where $\chi$ is the Euler character of the membrane. For a periodic web the genus is $g=F+1$, and we have $n_{F Z M}=4(4-\chi)$ 


\section{4 classification of BPS states}

The BPS states of $N=4 S U\left(N_{c}\right)$ SYM transform in irreducible representations of the $S L(2, \mathbb{Z})$ duality group. They can therefore be classified using $S L(2, \mathbb{Z})$ invariants. The simplest invariant is the number of independent non-vanishing $(p, q)$ charges.

The gluons in the Cartan subalgebra carry no charges and are therefore invariant under $S L(2, \mathbb{Z})$. Since they are massless, they are $1 / 2$ BPS states.

States carrying a single independent charge are also $1 / 2$ BPS states, but are generically massive. Note that there could still be two charges whose sum vanishes, as for the $((1,0),(-1,0)) W$-boson of $S U(3)$. All of these 1/2 BPS states can be obtained from the perturbative ones, namely from the $N_{c}\left(N_{c}-1\right) W$-bosons, by the action of the duality group. This will include all the monopoles and dyons carrying parallel and co-prime charges (p, q).

The $1 / 4$ BPS states are not related to the perturbative states by any $S L(2, \mathbb{Z})$ element, and therefore lie on separate $S L(2, \mathbb{Z})$ orbits [0]. As these states correspond to string webs, their classification will be similar, but not identical, to the classification of string webs. String webs with overlapping external strings give rise to multiple BPS states, depending on whether overlapping external strings end on the same D3-brane or not. Non-degenerate string webs with $E_{\text {ext }}$ external strings will give rise to $1 / 4$ BPS states carrying $E_{\text {ext }}-1$ independent $(p, q)$ charges, all of which are co-prime. On the other hand, degenerate string webs with overlapping external strings will give rise to these, as well as to 1/4 BPS states carrying less charges, some of which are non-co-prime, as in example 2 of section 3.2 (figure $1 \mathrm{~b}$ ). In counting 1/4 BPS states we must allow for non-co-prime charges, as long as there isn't a common divisor for all the charges. The latter case will correspond to a reducible string web, and therefore to a multi-particle state.

Consider for example 1/4 BPS states in $S U(3)$, or equivalently states carrying two independent charges in any $S U\left(N_{c}\right)$. Following the same steps as in section 2, we use $S L(2, \mathbb{Z})$ to transform one of the charges to $(k, 0)$. This leaves us with the second charge $(p, q)$. The charge $q$ is arbitrary, but $p$ is restricted to the interval $[0, q-1]$ as before. Unlike in the classification of string webs however, there are no further restrictions on $p$. In particular, neither $(p, q)$ nor $(-p-k,-q)$ are required to be co-prime. In summary, the $S L(2, \mathbb{Z})$ orbits of $1 / 4$ BPS states carrying two independent charges are classified by two arbitrary integers $k, q$ and an integer $p \in[0, q-1]$. It would be interesting to find a geometrical interpretation for $k$ and $q$, as was done for string webs in section 2.5. One could attempt to derive invariants by referring to the grid diagram, which for the above states is a triangle 1 . The obvious invariants are the number of internal points, and the number of edge points on each of the lines. These are however not independent, and non-trivially related to $k$ and $q$.

\footnotetext{
${ }^{10}$ This includes degenerate polygons which look like triangles, e.g. figure $2 \mathrm{~b}$.
} 


\section{5 curves of marginal stability and decay of BPS states}

Consider a BPS state in $N=4 S U\left(N_{c}\right)$ SYM with charges $\left(\left(p_{1}, q_{1}\right), \ldots,\left(p_{N_{c}-1}, q_{N_{c}-1}\right)\right)$. Assume the charge vector $\mathbf{p}$ is decomposed as $\mathbf{p}=\mathbf{p}^{\prime}+\mathbf{p}^{\prime \prime}$, and similarly for $\mathbf{q}$. The mass of the state in question satisfies a triangle inequality

$$
M_{(\mathbf{p}, \mathbf{q})} \leq M_{\left(\mathbf{p}^{\prime}, \mathbf{q}^{\prime}\right)}+M_{\left(\mathbf{p}^{\prime \prime}, \mathbf{q}^{\prime \prime}\right)} .
$$

There exists a subspace of the moduli space where the above inequality is saturated, and the BPS state is only marginally stable against decay into the two other BPS states.

This happens already for $1 / 2$ BPS states if $N_{c}>2$. Consider the $W$-bosons of $S U(3) \rightarrow$ $U(1)^{2}$. These carry charges $\pm((1,0),(0,0)), \pm((0,0),(1,0))$ and $\pm((1,0),(-1,0))$ under the two $U(1)$ 's, and are represented by fundamental strings between pairs of D3-branes. Their masses are therefore given by

$$
\begin{aligned}
M_{ \pm((1,0),(0,0))} & =\left|\vec{R}_{1}\right| \\
M_{ \pm((0,0),(1,0))} & =\left|\vec{R}_{2}\right| \\
M_{ \pm((1,0),(-1,0))} & =\left|\vec{R}_{1}-\vec{R}_{2}\right| .
\end{aligned}
$$

Consider the subspace of moduli space defined by $\vec{R}_{2}=c \vec{R}_{1}$, where $0<c<1$. This corresponds to choosing the Higgs VEV's in the two Cartan directions to be parallel. In this subspace

$$
M_{((1,0),(0,0))}=M_{((0,0),(1,0))}+M_{((1,0),(-1,0))},
$$

so the $((1,0),(0,0)) W$-boson is marginally stable against decay into the two others. One can similarly find marginal stability subspaces for the other two $W$-bosons, and more generally for all $1 / 2$ BPS states. Since these subspaces always have a boundary however, they do not divide moduli space into disjoint regions, and therefore the 1/2 BPS states are absolutely stable everywhere else.

The 1/4 BPS states exhibit marginal stability on subspaces of moduli space as well. Unlike their 1/2 BPS counterparts however, these subspaces are unbounded, and in fact divide the moduli space into two regions. As we will show using the string web picture, the $1 / 4$ BPS states exist only in one of the regions, and must therefore decay on the marginal stability subspace.

Consider what happens to the 3-string junction (figure 1a), with D3-brane boundaries, as one of the external strings degenerates, i.e. as one of the D3-branes moves toward the junction point (figure $5 \mathrm{a}, \mathrm{b}$ ). When it coincides with the junction, the string ending on it has vanishing length, and we are left with two open strings, which can now separate along the common D3-brane. If we continue to move the D3-brane in the same direction, we find that a BPS 3-string junction no longer exists, because the angle conditions (3.9) cannot be satisfied. 
We conclude that the corresponding $1 / 4$ BPS state $((1,0),(0,1))$ decays into two $1 / 2$ BPS states, e.g. $((1,1),(0,0))+((0,-1),(0,1))$, corresponding to the two open strings, when the D3-brane coincides with the junction point. The loci of the junction point allowed by supersymmetry defines a marginal stability subspace for the $1 / 4$ BPS state in question. The projection of this subspace on the plane of the string web gives a curve. Since supersymmetry fixes the angles between the strings, this curve is simply a circular arc between the ends of the other two strings, with a radius given by (label the two non-shrinking strings $a, b$ )

$$
r=\frac{\left|\vec{R}_{a}-\vec{R}_{b}\right|}{2 \sin \theta_{a b}} .
$$

By reflection symmetry, there is another arc on the other side. This gives a closed curve, which divides moduli space into a region inside the curve and a region outside the curve. The 1/4 BPS state exists only outside. This is reminiscent of the behavior of BPS states in Seiberg-Witten theory [36]. One could similarly consider shrinking one of the other two strings. This leads to two other curves of marginal stability.

This result generalizes to all string webs. For example, for the $1 / 4$ BPS states arising from the string web in figure 1d there are two marginal stability curves for each external string that shrinks (figure $5 \mathrm{c}, \mathrm{d}, \mathrm{e}$ ).

\section{Long BPS states and stable non-BPS states}

We have seen that as one moves in moduli space one can encounter a wall of marginal stability where BPS states seem either to decay, as in the case of $1 / 4$ BPS states in $S U(3)$, or not, as in the case of $1 / 2$ BPS states in $S U(3)$. We shall now see that there is yet another effect that can occur, namely that a BPS state can become non-BPS, but remain stable. The simplest example of this occurs in $S U(4)$.

Consider a planar string web with 4 or more external strings, for example figure $1 \mathrm{~d}$. The moduli are a set of $N_{c}-1$ vectors in $\mathbb{R}^{6}$. For $N_{c}=3$ they are always coplanar. For $N_{c}=4$, on the other hand, planar configurations occur only on a submanifold of co-dimension 4.

From section 3 we know that in planar configurations supersymmetric webs exist for any given charges and any $N_{c}$, as long as curves of marginal stability are not crossed. In particular, the state in figure $1 \mathrm{~d}$ will remain supersymmetric if we make small planar changes in the moduli.

Consider moving one of the D3-branes off the plane. Intuition about ropes suggests that the web deforms in such a way as to minimize its mass. Because each junction would be at mechanical equilibrium, the three strings that meet there should lie in one plane. Once the configuration is non-planar, the planes corresponding to the two junctions will no longer be parallel, and the configuration will break supersymmetry. For example, in figure 1d we would get two non-parallel fundamental strings, which break all the supersymmetries. 


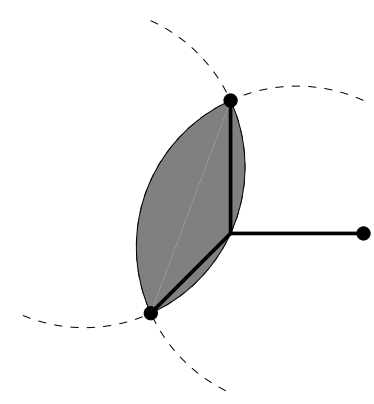

a

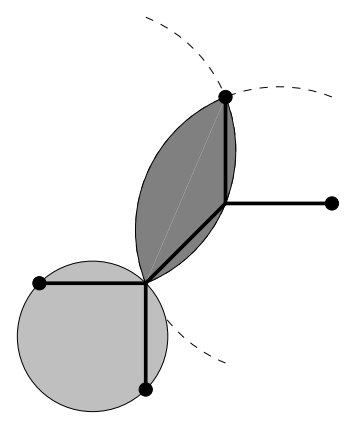

$\mathrm{c}$

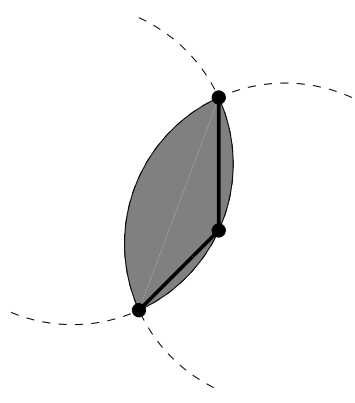

$\mathrm{b}$

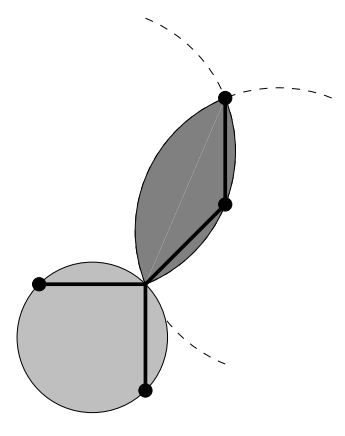

d

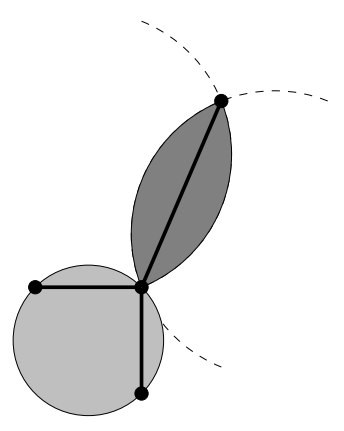

$\mathrm{e}$

Figure 5: Curves of marginal stability and decay of 1/4 BPS states. (a) 3-string junction decays into (b) two strings; (c) string web of fig. 1d decays first into (b) a string and a 3 -string junction, which proceeds to deacy into (c) two more strings.

This would seem to violate the stability of BPS states against changes in the moduli. Such stability is guaranteed for short (or medium) multiplets, since they cannot be smoothly connected to non-BPS states which come in long multiplets. The resolution is that the aforementioned BPS state is not in a medium representation. Indeed, from (3.22) we see that there are 16 FZM, only 12 of which correspond to broken supersymmetries. This means that the BPS state transforms as a long multiplet, and is therefore not protected against becoming non-BPS. We may call these states "long BPS multiplets" or "accidental BPS", as they occur only on the submanifold of planar configurations.

The long BPS state still satisfies the exact mass formula $M=Z_{+}$, since it is an algebraic consequence of unbroken supersymmetry. The non-planar webs are not BPS, but we may still look for extremal states of minimal mass $M_{e x}$ with the given charges. The classical mass of a non-planar web can be computed, and is found to be larger than the BPS bound. Furthermore, this mass may receive quantum corrections. If we assume that these states 
survive the transition to the quantum theory, and that BPS states are still absent, we would have a strict inequality

$$
M_{e x}>Z_{+} .
$$

One can choose some parameter of deviation from planarity $\phi$, and expand both quantities as a function of $\phi$ (assuming the function is regular at $\phi=0$ ),

$$
\begin{aligned}
M_{e x} & =m_{0}+m_{1} \phi+m_{2} \phi^{2} / 2+\cdots \\
Z_{+} & =m_{0}+z_{1} \phi+z_{2} \phi^{2} / 2+\cdots,
\end{aligned}
$$

where $m_{i}$ are the full non-perturbative coefficients of the mass of the non-BPS state. From (4.1) we conclude that

$$
\begin{aligned}
& m_{1}=z_{1} \\
& m_{2} \geq z_{2} .
\end{aligned}
$$

Since the planar configuration is invariant under reflections, $\phi$ may be chosen so that $M_{e x}, Z_{+}$ are even functions of $\phi$, and (4.3) is simplified.

\section{ACKNOWLEDGMENTS}

OB thanks Matthias Gaberdiel and Barton Zwiebach for helpful discussions. BK would like to thank Lenny Susskind; the hospitality at Tel-Aviv University and in particular Andreas Brandhuber, Jacob Sonnenschein and Shimon Yankielowicz; the hospitality of the Institute for Advanced Study, and in particular Amihay Hanany, Dan Kabat, Nathan Seiberg, Savdeep Sethi and Edward Witten; the hospitality of the workshop at the ITP, Santa Barbara and in particular Hirosi Ooguri; and discussions with Renata Kallosh, Arvind Rajaraman and Scott Thomas. OB is supported in part by the NSF under grant PHY-92-18167. BK is supported by NSF grant PHY-9219345.

\section{A String webs and the BPS formula}

We give here an alternative proof of the equality of the mass of a web and the BPS formula. Consider the $4 \mathrm{~d} N=4$ gauge theory, with $S U\left(N_{c}\right)$ gauge group and a complex coupling constant $\tau$. Its moduli space is parameterized by $N_{c} 6$-vectors $\vec{\Phi}_{a}=\Phi_{a}^{I}$, which satisfy $\sum_{a=1}^{N_{c}} \vec{\Phi}_{a}=0$, and which generically break the gauge group to $U(1)^{N_{c}-1}$. Consider a state with electric and magnetic charges $\left(p_{a}, q_{a}\right)$ under the various $U(1)$ 's, where $\sum p_{a}=\sum q_{a}=0$. This state can be realized as a string web with external strings carrying charges $\left(p_{a}, q_{a}\right)$. 
Define the 2 vectors $q_{a}^{A}=\left(\operatorname{Re}\left(p_{a}+\tau q_{a}\right), \operatorname{Im}\left(p_{a}+\tau q_{a}\right)\right)$. The central charge is determined in terms of a $2 \times 6$ matrix

$$
Z^{A I}=\sum_{a} q_{a}^{A} \Phi_{a}^{I}
$$

Consider first a 3-string web with no internal faces. Later we shall generalize to an arbitrary web. Choose the directions of the $\Phi$ coordinates in the plane of the web such that $\Delta\left(\Phi^{1}+\right.$ $\left.i \Phi^{2}\right)_{a}=\left(p_{a}+\tau q_{a}\right)$, as required by supersymmetry. We see that the charge vectors $q_{a}^{A}$ are parallel to the plane vectors $\Phi_{a}^{I}$ :

$$
\Phi_{a}^{I}=r_{a} q_{a}^{I} \quad(I=1,2)
$$

where $r_{a}$ are real and non-negative. The origin of the plane is chosen at the junction. The central charge matrix is now given by

$$
Z^{A I}=\sum_{a} q_{a}^{A} \Phi_{a}^{I}=\sum_{a} r_{a} q_{a}^{A} q_{a}^{I}
$$

which is a symmetric $2 \times 2$ matrix. In this case the formula (3.2) for the central charge simplifies to

$$
Z_{+}=|\operatorname{tr}(Z)|
$$

assuming $\operatorname{det}(Z) \geq 0$, which holds in our case. Computing $\operatorname{tr}(Z)$ we get

$$
\begin{aligned}
\operatorname{tr}(Z) & =\sum_{i, I} r_{i} \cdot q_{i}^{I} q_{i}^{I} \\
& =\sum_{i}|\vec{q}||\vec{\Phi}|=m_{w e b}
\end{aligned}
$$

After observing that if a web exists its mass equals the central charge, let us show that such a web can always be found, unless a marginal stability wall is crossed. To show that we will consider an existing web, which is not at marginal stability, and we will show that for any small change in the location of one of the D3-branes a web still exists. It is enough to consider a single D3-brane. If we move it along the string that is attached to it, no change is required in the web. If we move the D3-brane transverse to the string attached to it, we have one rotational degree of freedom that allows us to solve for the web. This is the angle defining the directions of the $\Phi^{1,2}$ axes.

To generalize for webs of arbitrary $N_{c}$ with possible internal faces, we first use the bosonic zero modes to shrink all internal faces. We are left with a "tree" like web. We set the origin at one of the vertices, and transform (A.1) from a sum over external strings, to a sum over all strings

$$
Z^{A I}=\sum_{a=1}^{E_{\text {ext }}} q_{a}^{A} \Phi_{a}^{I}=\sum_{a=1}^{E_{\text {ext }}} q_{a}^{A} \sum_{i \in a} \Phi_{i}^{I}=\sum_{i=1}^{E} q_{i}^{A} \Phi_{i}^{I}
$$


where the notation $i \in a$ means that the string $i$ is on the route from the origin to the external string $a$, and it is well defined for a tree web. Charge conservation at vertices is used in the last equality. At this point the previous argument can be repeated.

\section{References}

[1] J. Polchinski, "Dirichlet branes and Ramond-Ramond charges", Phys. Rev. Lett. 75 (1995) 4724, hep-th/9510017.

[2] For a review see: A. Giveon and D. Kutasov, "Brane dynamics and gauge theory", hep-th/9802067.

[3] A.A. Tseytlin, "Self-duality of Born-Infeld action and Dirichlet 3-brane of type IIB superstring theory", Nucl. Phys. B469 (1996) 51, hep-th/9602064; M.B. Green and M. Gutperle, "Comments on three-branes", Phys. Lett. B377 (1996) 28, hep-th/9602077.

[4] A. Sen, "Dyon-monopole bound states, selfdual harmonic forms on the multi-monopole moduli space, and $S L(2, \mathbb{Z})$ invariance in string theory", Phys. Lett. B329 (1994) 217, hep-th/9402032.

[5] For a review see: J.P. Gauntlett, "Duality and supersymmetric monopoles", Nucl.Phys.Proc.Suppl. 61A (1998) 137, hep-th/9705025.

[6] J. H. Schwarz, "An SL(2,Z) multiplet of Type IIB superstrings," Phys. Lett. B360 (1995) 13-18, hep-th/9508143.

[7] E. Witten, "Bound states of strings and p-branes", Nuc. Phys. B460 (1996) 335, hep-th/9510135.

[8] A. Sen, "F theory and orientifolds", Nucl. Phys. B475 (1996) 562, hep-th/9605150.

[9] A. Sen, "BPS states on a three brane probe", Phys. Rev. D55 (1997) 2501, hep-th/9608005.

[10] O. Aharony, J. Sonnenschein, S. Yankielowicz, "Interactions of strings and D-branes from M theory", Nucl. Phys. B474 (1996) 309-322, hep-th/9603009.

[11] J. H. Schwarz, "Lectures on superstring and $m$ theory dualities: Given at ictp spring school and at tasi summer school", Nucl. Phys. Proc. Suppl. 55B (1997) 1, hep-th/9607201. 
[12] O. Aharony and A. Hanany, "Branes, superpotentials and superconformal fixed points", Nucl. Phys. B504 (1997) 239, hep-th/9704170.

[13] M.R. Gaberdiel, B. Zwiebach, "Exceptional groups from open strings", hep-th/9709013.

[14] O. Aharony, A. Hanany, and B. Kol, "Webs of (p,q) five-branes, five-dimensional field theories and grid diagrams", JHEP 01 (1998), hep-th/9710116.

[15] K. Dasgupta and S. Mukhi, "BPS nature of 3-string junctions", hep-th/9711094.

[16] A. Sen, "String network", hep-th/9711130.

[17] S.J. Rey and J.T. Yee, "BPS dynamics of triple $(p, q)$ string junction", hep-th/9711202.

[18] M. Krogh and S. Lee, "String network from M-theory", hep-th/9712050.

[19] Y. Matsuo and K. Okuyama, "BPS condition of string junction from M-theory", hep-th/9712070.

[20] B. Kol and J. Rahmfeld, "BPS spectrum of five-dimensional field theories, (p,q) webs and curve counting", hep-th/9801067.

[21] O. Bergman, "Three pronged strings and 1/4 BPS states in $N=4$ super-Yang-Mills theory", hep-th/9712211.

[22] O. Bergman and A. Fayyazuddin, "String junctions and BPS states in Seiberg-Witten theory", hep-th/9802033.

[23] A. Mikhailov, N. Nekrasov, S. Sethi, "Geometric realizations of BPS states in N=2 theories", hep-th/9803142.

[24] O. Bergman and A. Fayyazuddin, "String junction transitions in Seiberg-Witten theory", to appear.

[25] Y. Imamura, "E(8) flavor multiplets", hep-th/9802189.

[26] E. Witten, "Phase transitions in M theory and F theory", Nucl. Phys. B471 (1996) 195-216, hep-th/9603150.

[27] B. Kol, "5-d field theories and M theory", hep-th/9705031.

[28] N. C. Leung and C. Vafa, "Branes and toric geometry", hep-th/9711013. 
[29] E. Witten and D. Olive, "Supersymmetry algebras that include topological charges", Phys. Lett. 78B (1978) 97.

[30] A. Sen, "Stable non-BPS states in string theory", hep-th/9803194.

[31] K. Hashimoto, H. Hata, N. Sasakura, "Three - string junction and BPS saturated solutions in SU(3) supersymmetric Yang-Mills theory", hep-th/9803127; T. Kawano and K. Okuyama, "String network and 1/4 BPS states in $\mathrm{N}=4 \mathrm{SU}(\mathrm{N})$ supersymmetric Yang-Mills theory", hep-th/9804139.

[32] S. Katz, A. Klemm, C. Vafa, "Geometric engineering of quantum field theories", Nucl. Phys. B497 (1997) 173-195, hep-th/9609239.

[33] C. G. Callan and L. Thorlacius, "World sheet dynamics of string junctions", hep-th/9803097.

[34] M.R. Gaberdiel and B. Zwiebach, private communication.

[35] H. Osborn, "Topological charges for $\mathrm{N}=4$ supersymmetric gauge theories and monopoles of spin 1", Phys. Lett. B83 (1979) 321; C. Fraser and T. Hollowood, "Semiclassical quantization in $\mathrm{N}=4$ supersymmetric Yang-Mills theory and duality", Phys. Lett. B402 (1997) 106, hep-th/9704011.

[36] N. Seiberg and E. Witten, "Electric-magnetic duality, monopole condensation, and confinement in N=2 supersymmetric Yang-Mills theory", Nucl. Phys. B426 (1994) 19, hep-th/9407087; "Monopoles, duality and chiral symmetry breaking in $\mathrm{N}=2$ supersymmetric QCD", Nucl. Phys. B431 (1994) 484, hep-th/9408099. 